\title{
Critical shear stress produced by interaction of edge dislocation with nanoscale inhomogeneity
}

\author{
Q H FANG*, J M CHEN, Y W LIU and P H WEN ${ }^{\dagger}$ \\ Department of Mechanical Engineering, Hunan International Economics University, \\ Changsha 410205, P.R. China \\ ${ }^{\dagger}$ Department of Engineering, Queen Mary, University of London, London EI 4NS, UK
}

MS received 30 December 2008; revised 26 April 2009

\begin{abstract}
According to the Mott and Nabarro's model, the contribution to the critical shear stress of the material caused by the interaction between edge dislocations and nanoscale cylindrical inhomogeneities with interface stresses is obtained. The influence of the radius and the volume fraction of the inhomogeneity as well as the interface stresses on the critical shear stress is investigated. The important result is that, if the interface stress is considered, a maximum of the contribution to the critical shear stress produced by this interaction may be obtained when the radius of the inhomogeneity reaches a critical value.
\end{abstract}

Keywords. Edge dislocation; nanoscale inhomogeneity; interface stress.

\section{Introduction}

The elastic problem of dislocations interacting with dispersed particles or second phase inclusions is of considerable interest for understanding the physical behaviour of crystalline solids. Such investigation can provide information concerning certain strengthening and hardening mechanism in a number of alloys and composites (Nembach 1996). When a dislocation is moved by an applied stress on one of the slip planes of the matrix, it will encounter dispersed particles or second phase inclusions which provide barriers to dislocation motion and thus affect the flow stress of the material. In the literature a great number of possible strengthening methods had been discussed (Kelly and Nicholson 1971). The modulus mismatch strengthening is an important strengthening method which arises from different elastic moduli of the matrix and the inclusion. For this strengthening effect, the elementary interaction between a single inclusion and a dislocation on the nearby slip plane will be first considered which leads to an interaction force acting on the dislocation (Nembach 1996). Considering its importance, this interaction had received much attention during the last several decades (Dundurs and Mura 1964; Smith 1968; Hirth and Lothe 1982; Stagni and Lizzio 1983; Luo and Chen 1991; Xiao and Chen 2001; Wang and Sudak 2006; Wang et al 2007; Takahashi and Ghoniem 2008; etc). In addition, for the modulus mismatch strengthening method, Russell and Brown (1972) gave an approximate

*Author for correspondence (fangqh1327@tom.com) expression for the critically resolved shear stress of the materials strengthened by a modulus difference between the matrix and the precipitate. The force acting on the dislocation produced by the interaction between a spherical precipitate, whose shear modulus differs from the one of the matrix, and a dislocation was calculated numerically by Nembach (1983). From the maximum and the range of the obtained interaction force, the critically resolved shear stress was also derived.

Dislocation mobility plays a significant role in understanding of the strengthening mechanism of crystalline materials. The mobility of the dislocation is dependent on many factors, including composition of the materials, temperature and interface conditions of the inclusions. The interface boundary condition is one of the important factors. The interface of solids is a special region of very small thickness. Since the equilibrium lattice spacing in the interface is different from that in the bulk, the interface stress appears. For solids with large characteristic dimensions, the volume ratio of the interface region to the bulk material is very small. The effect of the interface stress is insignificant and can be rightfully neglected. However, for fine-scaled materials, such as nanocomposites, with a large ratio of the interface region to the bulk, the interface plays a very important role, and the contribution of the interface stress to the property of the materials is significant (Miller and Shenoy 2000). A general model for elastic isotropic solids incorporating surface/ interface stress was proposed by Gurtin and Murdoch (1975). Great effort has been made recently to understand some unusual phenomena related to surface/interface stress in nanostructured materials (Cammarata et al 2000; 
Sharma et al 2003; Duan et al 2005; Lim et al 2006; Chen and Dvorak 2006; Quang and He 2007 etc).

In the present paper, using the obtained complex potentials of the interaction between an edge dislocation and a nanoscale circular cylindrical inclusion with interface stress (Fang and Liu 2006), the interaction force acting on the edge dislocation along the glide direction is calculated. The contribution to the critical shear stress of the materials due to this interaction is derived by using the obtained interaction force and the Mott and Nabarro's (1940) model. The influence of the interface stress on the critical shear stress is examined. Although the model is formulated in terms of a cylindrical inclusion of infinite extent rather than the more appropriate spherical case, it is generally believed that the important features of the threedimensional problem are retained (Srolovitz et al 1983).

\section{Solution of critical shear stress}

Consider an infinite elastic medium with elastic properties, $\kappa_{2}$ and $\mu_{2}$, containing a circular nanoscale inhomogeneity of a radius, $R$, with elastic properties, $\kappa_{1}$ and $\mu_{1}$, where $\mu_{j}(j=1,2)$ is the shear modulus and $\kappa_{j}=3-4 v_{j}$ for plane strain state ( $v_{j}$ is the Poisson's ratio). A straightline edge dislocation with Burgers vector, $b_{r}$ (along dislocation glide direction) is assumed to be located inside the matrix at arbitrary point, $z_{0}=r_{0} e^{i \theta}$ and parallel to the axis of cylindrical inhomogeneity. The regions occupied by the inhomogeneity and the matrix will be denoted by $S^{+}$ and $S^{-}$, respectively.

As the present problem is a plane elastic one, following Sharma et al (2003), the displacement components are continuous on the interface and the stress boundary conditions on the interface can be summarized as

$$
\sigma_{r r 1}^{+}(t)-\sigma_{r r 2}^{-}(t)=-\sigma_{\theta \theta}^{0} / R
$$

and

$$
\sigma_{r \theta 1}^{+}(t)-\sigma_{r \theta 2}^{-}(t)=\left(\partial \sigma_{\theta \theta}^{0} / \partial \theta\right) / R,
$$

where $\sigma_{r r}$ and $\sigma_{r \theta}$ are stress components in polar coordinates system, $r$ and $\theta$, the superscripts + and - denote the boundary values of a physical quantity as $z$ approaches the interface from $S^{+}$and $S^{+}$, respectively. In addition, the subscripts 1 and 2 represent the regions $S^{+}$and $S^{-}$, and the superscript ' 0 ' denotes the interface region. An additional constitutive equation for the interface region is given as

$$
\sigma_{\theta \theta}^{0}=\left(2 \mu^{0}+\lambda^{0}\right) \varepsilon_{\theta \theta}^{0},
$$

where $\sigma_{\theta \theta}^{0}$ and $\varepsilon_{\theta \theta}^{0}$ denote interfacial stress and strain, $\mu^{0}$ and $\lambda^{0}$ are interfacial elastic constants. For a coherent interface, the interfacial strain, $\varepsilon_{\theta \theta}^{0}$, equals to the associated tangential strain in the abutting bulk materials. With a semi-coherent or incoherent interface, an additional measure of the interfacial strain is required (Cammarata et al 2000).
For the above problem, by using complex variable techniques of Muskhelishvili, the complex potential functions and the stress fields in the matrix and inhomogeneity regions can be obtained (Fang and Liu 2006). The image force acting on the edge dislocation along the Burgers vector direction (dislocation glide direction) can be derived from the stress fields, which is given as follows

$$
\begin{aligned}
& f_{r}=\frac{\mu_{2} b_{r}^{2}}{\pi\left(1+\kappa_{2}\right)}\left[\frac{r_{0} \Phi_{2}^{* \prime}\left(r_{0}\right)+\Psi_{2}^{*}\left(r_{0}\right)}{\bar{\gamma}_{2}}\right], \\
& \Phi_{2}^{*}\left(r_{0}\right)=\frac{\mu_{2}}{\mu_{1} \kappa_{2}} \sum_{k=1}^{+\infty} B_{-k}\left(r_{0}\right)^{-k} \\
& +\frac{1}{\kappa_{2}}\left[\frac{\gamma_{2}}{r_{0}-z^{*}}-\frac{\gamma_{2}}{r_{0}}-\frac{\bar{\gamma}_{2} z^{*}}{r_{0}\left(r_{0}-z^{*}\right)}\right], \\
& \Phi_{2}^{* \prime}\left(r_{0}\right)=-\frac{\mu_{2}}{\mu_{1} \kappa_{2}} \sum_{k=1}^{+\infty} k B_{-k}\left(r_{0}\right)^{-k-1} \\
& +\frac{1}{\kappa_{2}}\left[\frac{\gamma_{2}}{\left(r_{0}\right)^{2}}-\frac{\gamma_{2}}{\left(r_{0}-z^{*}\right)^{2}}+\frac{2 \bar{\gamma}_{2} z^{*}}{r_{0}\left(r_{0}-z^{*}\right)^{2}}\right] \text {, } \\
& \Psi_{2}^{*}\left(r_{0}\right)=\frac{R^{2}}{r_{0}^{2}}\left[\Phi_{2}^{*}\left(r_{0}\right)-r_{0} \Phi_{2}^{*^{\prime}}\left(r_{0}\right)\right] \\
& +\frac{R^{2}}{r_{0}^{2}}\left\{\frac{\mu_{2} \kappa_{1}}{\mu_{1}}\left[\sum_{k=1}^{+\infty} \bar{A}_{k}\left(\frac{R^{2}}{r_{0}}\right)^{k}-\frac{\mu_{1}\left(1+\kappa_{2}\right)}{\mu_{2} \kappa_{1}+\mu_{1}(1+b)} \frac{\bar{\gamma}_{2}}{\bar{r}_{0}}\right]\right. \\
& -\frac{\kappa_{2} \bar{\gamma}_{2} r_{0}}{R^{2}-r_{0}^{2}}-\frac{\mu_{2}\left(1+\kappa_{1}\right)(1+b)}{\mu_{2} \kappa_{1}+\mu_{1}(1+b)} A_{0} \\
& \left.+\frac{\gamma_{2}\left(r_{0}^{2}-R^{2}\right)}{R^{2} r_{0}}+\frac{2 \bar{\gamma}_{2} r_{0}}{R^{2}}\right\}-\frac{2 \bar{\gamma}_{2}}{r_{0}}-\frac{\gamma_{2}}{r_{0}}
\end{aligned}
$$

where $z^{*}=R^{2} / r_{0}, \quad \gamma_{2}=\frac{-\mu_{2} i b_{x}}{\pi\left(1+\kappa_{2}\right)}, \quad a=\frac{2 \mu^{0}+\lambda^{0}}{4 R \mu_{1}} \quad$ and $b=\frac{2 \mu_{1}\left(2 \mu^{0}+\lambda^{0}\right)}{4 R \mu_{1}\left(\lambda_{1}+\mu_{1}\right)}$.

In addition, the expressions of the coefficients $A_{k}$ and $B_{-k}$ can be expressed as

$$
\begin{aligned}
& A_{0}=\frac{c_{1}}{\left(c_{2}\right)^{2}-1}\left(\frac{c_{2} \bar{\gamma}_{2}}{\bar{z}_{0}}+\frac{\gamma_{2}}{z_{0}}\right), \\
& B_{0}=-\bar{A}_{0}=\frac{c_{1}}{1-\left(c_{2}\right)^{2}}\left(\frac{\bar{\gamma}_{2}}{\bar{z}_{0}}+\frac{c_{2} \gamma_{2}}{z_{0}}\right),
\end{aligned}
$$




$$
\begin{aligned}
A_{k}= & -\frac{\left(1+\kappa_{2}\right)}{c_{3}} \frac{\gamma_{2}}{\left(z_{0}\right)^{k+1}}+\frac{c_{4}}{c_{3}}\left[\bar{\gamma}_{2}\left(\bar{z}^{*}\right)^{k-1} R^{-2 k}-\bar{\gamma}_{2} R^{-2 k} \delta_{1 k}\right. \\
& \left.-\frac{\gamma_{2} \bar{z}^{*}\left(\bar{z}_{0}-\bar{z}^{*}\right) R^{-2 k}(k-1)\left(\bar{z}^{*}\right)^{k-2}}{z_{0}}\right] k \geq 1 \\
B_{-k}= & -\frac{c_{6}}{c_{5}} \frac{\bar{\gamma}_{2} R^{2 k}}{\left(\bar{z}_{0}\right)^{k+1}}-\frac{1+\kappa_{2}}{c_{5} \kappa_{2}} \\
& {\left[\begin{array}{l}
\left.\gamma_{2}\left(z^{*}\right)^{k-1}-\gamma_{2} \delta_{1 k}-\frac{\bar{\gamma}_{2} z^{*}\left(z_{0}-z^{*}\right)(k-1)\left(z^{*}\right)^{k-2}}{\bar{z}_{0}}\right]
\end{array}\right.}
\end{aligned}
$$

$k \geq 1$,

where $c_{1}=\frac{\mu_{1}\left(1+\kappa_{2}\right)}{\mu_{2} \kappa_{1}+\mu_{1}(1+b)}, c_{2}=\frac{\mu_{2}-\mu_{1}(1+b)}{\mu_{2} \kappa_{1}+\mu_{1}(1+b)}$,

$$
\begin{aligned}
& c_{3}=1+(a+b)(k+1)+\frac{\mu_{2} \kappa_{1}}{\mu_{1}}+\frac{a(a+b)(1+k)(1-k)}{1-a+k a+\mu_{2} /\left(\kappa_{2} \mu_{1}\right)}, \\
& c_{4}=\frac{\left(1+\kappa_{2}\right) a(1+k)}{\kappa_{2}\left[1-a+k a+\mu_{2} /\left(\kappa_{2} \mu_{1}\right)\right]}, \\
& c_{5}=1+a(k-1)+\frac{\mu_{2}}{\mu_{1} \kappa_{2}}+\frac{a(a+b)(1+k)(1-k)}{1+(a+b)(k+1)+\mu_{2} \kappa_{1} / \mu_{1}}, \\
& c_{6}=\frac{\left(1+\kappa_{2}\right)(a+b)(1-k)}{1+(a+b)(k+1)+\mu_{2} \kappa_{1} / \mu_{1}}
\end{aligned}
$$

and $\delta_{i j}$ is the Kronecker delta.

The critical shear stress is defined as the shear stress necessary to make dislocation glide over macroscopic distance. In inhomogeneity-strengthened materials, the applied stress must be high enough to overcome the interaction forces due to the interaction between inhomogeneities and dislocations. In a real material the total critical shear stress comprises of different contributions: that due to inhomogeneities; that due to atoms solved in the matrix; and so on. In the present paper, the contribution to the critical shear stress caused by the interaction between edge dislocations and inhomogeneities is only considered.

According to the work of Mott and Nabarro (1940), consider the composites containing $N$ inclusions per unit area, each of inclusions with radius $R$. The average distance of the edge dislocation in the matrix from the nearest of them is half the distance between inclusions or $r_{0}=\left(N^{-1 / 2}\right) / 2$. Thus if $C=N \pi R^{2}$ is the volume fraction of inclusions, the mean force $f_{r m}$ acting on the edge dislocation can be obtained

$$
f_{r m}=\frac{\mu_{2} b_{r}^{2}\left(\Pi_{1}+\Pi_{2}\right)}{\pi\left(1+\kappa_{2}\right)}
$$

$$
\begin{array}{r}
\Pi_{1}=\frac{4 C}{\pi}\left(\Pi_{3}-\Pi_{2}\right)-\frac{4 C}{\pi} \frac{\mu_{2} \kappa_{1}}{\mu_{1}} \sum_{k=1}^{+\infty}\left\{\frac{1+\kappa_{2}}{c_{3}}\left(\frac{4 C}{\pi}\right)^{k} \frac{2 \sqrt{C}}{R \sqrt{\pi}}\right. \\
\left.+\frac{c_{4}}{c_{3}}\left[\left(\frac{4 C}{\pi}\right)^{k-1}-\delta_{1 k}+(k+1)\left(\frac{4 C}{\pi}\right)^{k-1}\left(1-\frac{4 C}{\pi}\right)\right] \frac{2 \sqrt{C}}{R \sqrt{\pi}}\right\} \\
-\frac{4 C}{\pi} \frac{\mu_{2} \kappa_{1}\left(1+\kappa_{2}\right)}{\mu_{2} \kappa_{1}+\mu_{1}(1+b)} \frac{2 \sqrt{C}}{R \sqrt{\pi}}-\frac{4 C}{\pi}\left[\frac{2 \kappa_{2} \sqrt{C \pi}}{(4 C-\pi) R}\right. \\
\left.+\frac{\mu_{2}\left(1+\kappa_{1}\right)(1+b)}{\mu_{2} \kappa_{1}+\mu_{1}(1+b)} \frac{c_{1}}{1+c_{2}} \frac{2 \sqrt{C}}{R \sqrt{\pi}}+\left(\frac{\pi}{4 C}-2\right) \frac{2 \sqrt{C}}{R \sqrt{\pi}}-\frac{2 \sqrt{\pi}}{R \sqrt{C}}\right],
\end{array}
$$

$$
\begin{aligned}
\Pi_{2}= & \frac{1}{\kappa_{2}}\left[\frac{2 \pi^{3 / 2} C^{1 / 2}+8 \pi^{1 / 2} C^{3 / 2}}{R(\pi-4 C)^{2}}-\frac{2 \sqrt{C}}{R \sqrt{\pi}}\right] \\
& -\frac{\mu_{2}}{\mu_{1} \kappa_{2}} \sum_{k=1}^{+\infty}\left\{\frac { ( 1 + \kappa _ { 2 } ) k } { c _ { 5 } \kappa _ { 2 } } \left[\left(\frac{4 C}{\pi}\right)^{k-1}-\delta_{1 k}\right.\right. \\
& \left.\left.+\left(\frac{4 C}{\pi}\right)^{k-1}\left(1-\frac{4 C}{\pi}\right)\right] \frac{2 \sqrt{C}}{R \sqrt{\pi}}-\frac{c_{6}}{c_{5}}\left(\frac{4 C}{\pi}\right)^{k} \frac{2 k \sqrt{C}}{R \sqrt{\pi}}\right\}, \\
\Pi_{3}= & \frac{1}{\kappa_{2}}\left[\frac{2 \sqrt{C}}{R \sqrt{\pi}}-\frac{2 \pi C^{1 / 2}+8 C^{3 / 2}}{R \pi^{1 / 2}(\pi-4 C)}\right]^{k-1}-\delta_{1 k} \\
+ & \left.\frac{\mu_{2}}{\mu_{1} \kappa_{2}} \sum_{k=1}^{+\infty}\left\{\frac{\left(1+\kappa_{2}\right)}{c_{5} \kappa_{2}}\left[\left(\frac{4 C}{\pi}\right)^{k-1}\right)^{k-1}\left(1-\frac{4 C}{\pi}\right)\right] \frac{2 \sqrt{C}}{R \sqrt{\pi}}-\frac{c_{6}}{c_{5}}\left(\frac{4 C}{\pi}\right)^{k} \frac{2 \sqrt{C}}{R \sqrt{\pi}}\right\} \\
+ & \left(\frac{4 C}{\pi}\right)^{k}
\end{aligned}
$$

Referring to the work of Nembach (1996), the critical shear stress caused by this elastic interaction (or the contribution to the critical shear stress of the material) can be derived from the mean force in (8).

$$
\Delta \tau_{c}=\frac{\mu_{2} b_{r}\left[\Pi_{1}(R, C)+\Pi_{2}(R, C)\right]}{\pi\left(1+\kappa_{2}\right)} .
$$

\section{Numerical examples and discussion}

The influence of the radius, $R$, of the nanoscale inhomogeneity and the volume fraction of the nanoscale inhomogeneities, $C$, on the contribution to the critical shear stress caused by the elastic interaction can be inves- 
tigated through (13). In subsequent numerical results we take Burgers vector, $b_{r}=0.3 \mathrm{~nm}$ and $v_{1}=v_{2}=0.3$. In addition, we define the parameters $\alpha=\mu^{0} / \mu_{1}$ and $\beta=\lambda^{0} / \mu_{1}$ ( $\mu^{0}$ and $\lambda^{0}$ are interfacial elastic constants $)$, the ratio of the shear moduli of two bulk solids $\eta=\mu_{2} / \mu_{1}$. The normalized critical shear stress, $\Delta \tau_{c 0}$, is defined as

$$
\Delta \tau_{c 0}=\left(\Delta \tau_{c} * 10^{4}\right) / \mu_{2} .
$$

For the description of the interface properties one needs the interfacial elastic constants, $\mu^{0}$ and $\lambda^{0}$. Unfortunately, they are currently unavailable in the literature. Miller and Shenoy (2000) computed free surface for aluminum and silicon by the embedded atom method (EAM), and indicated that the surface properties can be either positive or negative, depending upon crystallographic orientation. According to their results, the absolute value of $\mu^{0} / \mu$ and $\lambda^{0} / \mu$ is nearly $0 \cdot 1 \mathrm{~nm}$ (here $\mu$ is the shear modulus of the bulk solid).

The critical shear stress, $\Delta \tau_{c 0}$, is plotted as a function of the radius, $R$ in figure 1 with different values of $\alpha$ and $\beta$ for the relative shear modulus, $\eta=\mu_{2} / \mu_{1}=0.85$ and the volume fraction, $C=0 \cdot 1$. It can be found from figure 1 that the contribution to the critical shear stress, $\Delta \tau_{c 0}$, will increase with the decrease of the inhomogeneity radius, $R$, if $\alpha=\beta=0 \mathrm{~nm}$ or $\alpha=\beta=0.15 \mathrm{~nm}$. However, if we take $\alpha=\beta=-0.15 \mathrm{~nm}$, the contribution to the critical shear stress, $\Delta \tau_{c 0}$, increases first, and then decreases with the decrease of the inhomogeneity radius, $R$. In this case, when the volume fraction of the nanoscale inhomogeneities keeps a constant, there is a critical value of the inhomogeneity radius, $R$, to determine the maximal contribution to the critical shear stress of the material. The phenomenon cannot be predicted by the classical solutions without considering the interface stress. The result indicates that an optimal value of the radius, $R$, of the inhomogeneity with interface stress may exist to obtain the best strengthening effect for composite materials under certain conditions.

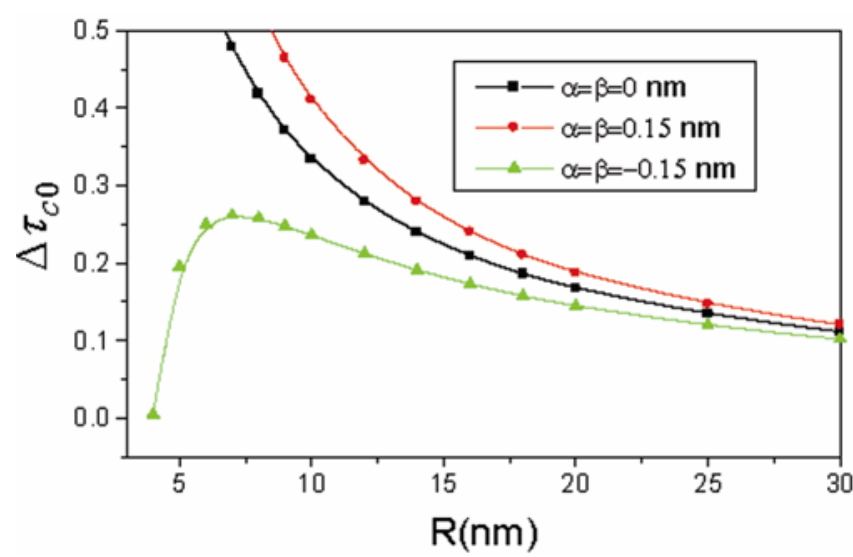

Figure 1. The critical shear stress, $\Delta \tau_{c 0}$, as a function of the radius, $R$, with different values of $\alpha$ and $\beta$ for $\eta=0.85$ and $C=0 \cdot 1$
The variation of the normalized critical shear stress, $\Delta \tau_{c 0}$, with respect to the volume fraction, $C$, is depicted in figure 2 with different values of $\alpha$ and $\beta$ for $R=10 \mathrm{~nm}$ and $\eta=\mu_{2} / \mu_{1}=0 \cdot 85$. An interesting result from figure 2 is that the contribution to the critical shear stress will increase first, and then decreases with the increment of the volume fraction $C$ if $\alpha=\beta=-0.15 \mathrm{~nm}$ and the radius of the nanoscale inhomogeneity keeps a constant. There also exists a critical volume fraction of the inhomogeneities, $C$, to obtain the maximal contribution to the critical shear stress of the material.

The critical shear stress, $\Delta \tau_{c 0}$, is plotted as a function of radius $R$ in figure 3 with different values of $\alpha$ and $\beta$ and the relative shear modulus $\eta=\mu_{2} / \mu_{1}$ for the volume fraction $C=0 \cdot 1$. It is seen that, if the interface stresses are negative ( $\alpha=\beta=-0.15 \mathrm{~nm})$ and the nanoscale inhomogeneity is stiffer than the matrix $\left(\eta=\mu_{2} / \mu_{1}=0.85\right)$, the contribution to the critical shear stress of the material caused by the interaction of edge dislocations with the inhomogeneities is positive first, and then becomes negative with the decrease of radius $R$. This result shows that

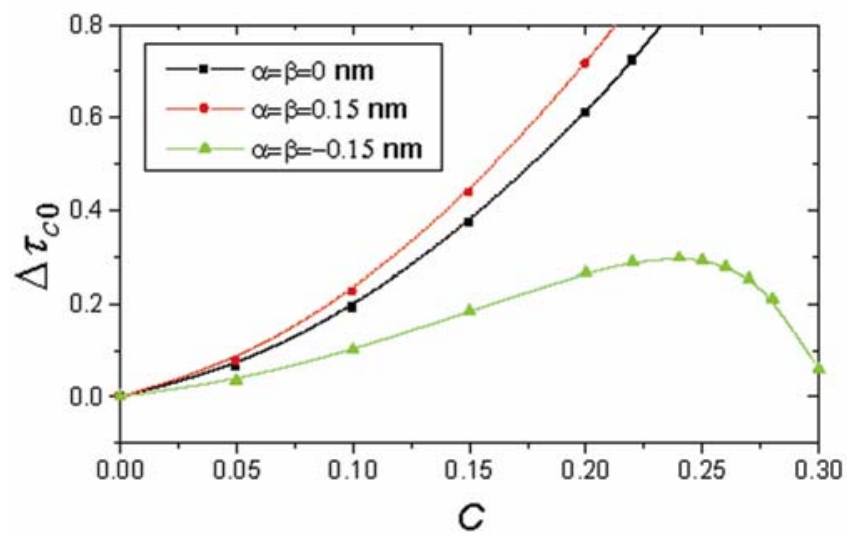

Figure 2. The critical shear stress, $\Delta \tau_{c 0}$, as a function of the volume fraction, $C$, with different values of $\alpha$ and $\beta$ for $\eta=0.85$ and $R=10 \mathrm{~nm}$.

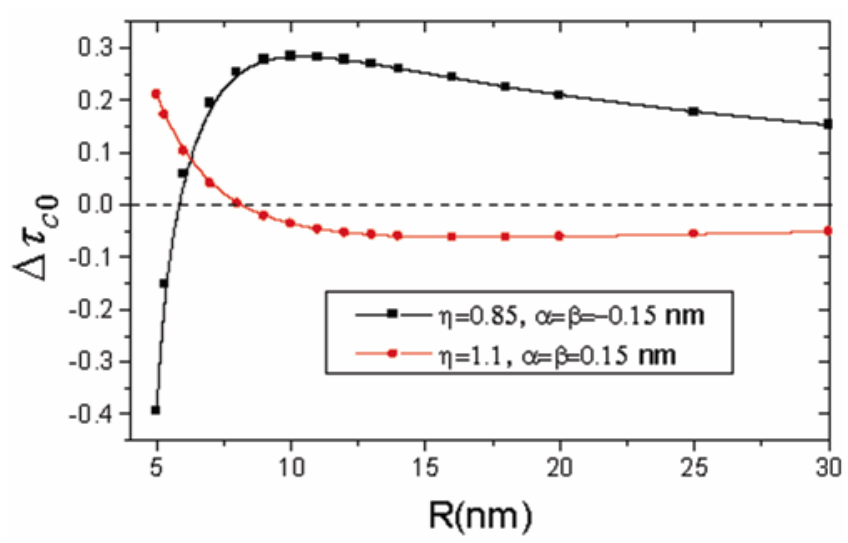

Figure 3. The critical shear stress, $\Delta \tau_{c 0}$, as a function of the radius, $R$, with different values of $\alpha$ and $\beta$ and the relative shear modulus $\eta=\mu_{2} / \mu_{1}$ for $C=0 \cdot 1$. 
the material may be softened by the stiff nanoscale inhomogeneity when the radius of the inhomogeneity is very small and the interface stresses are considered. Similarly, if the interface stresses are positive $(\alpha=\beta=0.15 \mathrm{~nm})$ and the nanoscale inhomogeneity is softer than the matrix, the contribution to the critical shear stress is negative first, and then becomes positive with the decrease of the radius $R$, which also shows that the material can be strengthened by the soft nanoscale inhomogeneity under certain conditions.

\section{Conclusions}

Based on the Mott and Nabarro's model, the contribution to the critical shear stress produced by the interaction between edge dislocations and nanoscale cylindrical inhomogeneities with interface stresses is derived. The effect of the interface stresses on the critical shear stress is examined. The results indicate that, if the volume fraction of the nanoscale inhomogeneities keeps a constant and the interface stresses are considered, there is a critical value of radius of the inhomogeneity to determine the maximal contribution to the critical shear stress of the material under some conditions. In addition, if the radius of the inhomogeneity keeps a constant, there also exists a critical volume fraction to obtain the maximal contribution to the critical shear stress caused by this interaction. It is also found that, when the interface stresses are considered, the composite materials can be strengthened by the soft nanoscale inhomogeneity under certain conditions.

\section{Acknowledgements}

The authors would like to deeply appreciate the support by the NNSFC (50801025, 50634060 and 10872065), China Postdoctoral Science Foundation funded project
(20080430151) and the Ph.D. Programs Foundation of Ministry of Education of China (200805321015).

\section{References}

Cammarata R C, Sieradzki K and Spaepen F $2000 \mathrm{~J}$. Appl. Phys. 871227

Chen T and Dvorak G J 2006 Appl. Phys. Lett. 88211912

Duan H L, Wang J, Huang Z P and Karihaloo B L 2005 J. Mech. Phys. Solids $\mathbf{5 3} 1574$

Dundurs J and Mura T 1964 J. Mech. Phys. Solids 12177

Fang Q H and Liu Y W 2006 Acta Mater. 544213

Gurtin M E and Murdoch A I 1975 Arch. Rat. Mech. Anal. 57 291

Hirth J P and Lothe J 1982 Theory of dislocations (New York: John Wiley) 2nd edn

Kelly A and Nicholson R B 1971 Strengthening methods in crystals (Amsterdam: Elsevier)

Lim C W, Li Z R and He L H 2006 Int. J. Solids Struct. 43 5055

Luo H A and Chen Y 1991 J. Appl. Mech. 5875

Miller R E and Shenoy V B 2000 Nanotechnology 11 139

Mott N F and Nabarro F R N 1940 Proc. Phys. Soc. 5286

Nembach E 1983 Phys. Status Solidi (a) 78571

Nembach E 1996 Particle strengthening of metals and alloys (New York: Wiley)

Quang H L and He Q C 2007 J. Mech. Phys. Solids 551899

Russell K C and Brown L M 1972 Acta Metall. 20969

Sharma P, Ganti S and Bhate N 2003 Appl. Phys. Lett. 82535

Smith E 1968 Int. J. Eng. Sci. 6129

Srolovitz D J, Petkoviv-Luton R A and Luton M J 1983 Acta Metall. 312151

Stagni L and Lizzio R 1983 Appl. Phys. A30 217

Takahashi A and Ghoniem N M 2008 J. Mech. Phys. Solids 56 1534

Xiao Z M and Chen B J 2001 Int. J. Solids Struct. 382533

Wang X and Sudak L J 2006 J. Appl. Mech. 73206

Wang X, Pan E and Roy A K 2007 J. Mech. Phys. Solids 55 2717 